\title{
Reservoir host studies of Lloviu virus: first isolation, sequencing and serology in Schreiber's bats in Europe
}

Gábor Kemenesi*^ ${ }^{\star}$, Gábor Endre Tóth ${ }^{\mathrm{d}}$, Martin Mayora-Neto, Simon Scott, Nigel Temperton,

Edward Wright, Elke Mühlberger, Adam J. Hume, Brigitta Zana, Sándor András Boldogh,

Tamás Görföl, Péter Estók, Zsófia Lanszki, Balázs A. Somogyi, Ágnes Nagy, Csaba István

Pereszlényi, Gábor Dudás, Fanni Földes, Kornélia Kurucz, Mónika Madai, Safia Zeghbib,

Piet Maes, Bert Vanmechelen, Ferenc Jakab

Affiliations:

G.K., G.E.T., B.Z., T.G., Z.L., B.A.S., F.F., K.K., M.M., S.Z. \& F.J.: National Laboratory of Virology, Szentágothai Research Centre, University of Pécs, Ifjúság útja 20., Pécs, H-7624, Hungary. ORCID - G.K.: 0000-0001-9775-3065, B.Z.: 0000-0002-3503-8901, T.G.: 00000002-1910-4024, Z.L.: 0000-0003-3116-4633, B.A.S.: 0000-0002-6006-9873, F.F.: 00000002-4693-9406, K.K.: 0000-0001-6190-1265, F.J.: 0000-0002-3913-0430

M.M.-N., S.S. \& N.T.: - Viral Pseudotype Unit, Medway School of Pharmacy, Universities of Kent \& Greenwich, Chatham Maritime, Kent, ME4 4TB, UK. ORCID - S.S.: 0000-00028290-0461, M.M.-N.: 0000-0002-5331-5554, N.T.:0000-0002-7978-3815

E.W. - Viral Pseudotype Unit, School of Life Sciences, University of Sussex, Falmer, Sussex, BN1 9RH, UK. ORCID - E.W.: 0000-0001-7041-5138

E.M. \& A.J.H.: Department of Microbiology, Boston University School of Medicine, Boston, MA 02118, USA. ORCID - E.M.: 0000-0003-3547-9376, A.J.H.: 0000-0001-8454-3472

S.A.B.: Aggtelek National Park Directorate, Tengerszem oldal 1., Jósvafö, H-3758, Hungary. ORCID - S.A.B.: 0000-0001-5887-5238

P.E.: Department of Zoology, Eszterházy Károly University, Eszterházy tér 1., Eger, H-3300, Hungary. ORCID - E.P.: 0000-0001-5303-0340

Á.N, C.I.P. \& G.D.: Medical Centre, Hungarian Defence Forces, Róbert Károly Krt. 44., Budapest, H-1134, Hungary

P.M, B.V: KU Leuven, Rega Institute, Department of Microbiology, Immunology and Transplantation, Laboratory of Clinical and Epidemiological Virology, Leuven, Belgium.

${ }^{\mathrm{d}}$ These authors contributed equally to the manuscript

*Corresponding author: G.K. 
Introductory

Filoviruses are prime examples of emerging human pathogens that are transmitted to humans by zoonotic spillover events. Since their initial discovery, filovirus outbreaks have occured with increasing frequency and intensity. There is an urgent need to better understand their enzootic ecology and pathogenic potential, given recent zoonotic virus spillover events including the 2013-2016 West African Ebola virus (EBOV) epidemic. Several novel filoviruses have been discovered with a markedly wider geographic distribution than previously described. One of these novel filoviruses, Lloviu virus (LLOV), was first identified in 2002 in Schreiber's bats (Miniopterus schreibersii) in Spain, Portugal, and southern France. Subsequently, in 2016, LLOV was detected during the passive monitoring of bats in Hungary.

Here we report the first isolation of infectious Lloviu virus; from the blood of an asymptomatic Schreiber's bat, subsequently cultivated in the Miniopterus sp. kidney cell line SuBK12-08. We also show that LLOV is able to infect monkey and human cells, suggesting that LLOV might have spillover potential. We performed a multi-year surveillance of LLOV and detected LLOV RNA in both deceased and asymptomatic live animals as well as in coupled ectoparasites from the families Nycteribiidae and Ixodidae. We present data on the natural infection and seropositivity of Schreiber's bats and provide novel LLOV genomic sequence information from a bat host and an arthropod parasite. Our data support the role of bats, specifically Miniopterus schreibersii as natural reservoirs for the LLOV filovirus in Europe. We also suggest that batassociated parasites might play a role in the natural ecology of filoviruses in temperate climate regions compared to filoviruses in the tropics. These results raise the possibility of cross-species transmission events via bat-human interactions. 


\section{Main}

Some members of the Filoviridae family are known to cause severe disease and frequent death in humans (e.g. ebolaviruses and marburgviruses), while there are other members with no known pathogenicity to humans (e.g. Reston virus) ${ }^{1}$. Among filoviruses, ebolaviruses have received the most attention, both by public health experts and the public, mainly due to the multiple documented human outbreaks, particularly the West African Ebola virus (EBOV) disease outbreak in 2013-2016 and the recent outbreaks in the Democratic Republic of the Congo and Guinea ${ }^{2,3,4}$. The first direct evidence pointing towards bats as natural reservoirs for EBOV was published in 2005, when RNA and virus-specific antibodies were detected in three species of fruit bats captured in Gabon and the Democratic Republic of the Congo 5 . To date, direct isolation of the infectious virus from a specific bat species has only been achieved in the case of Marburg virus (MARV), which leaves several open questions regarding the natural reservoir host for other filoviruses ${ }^{6}$. During the past few years, increased scientific focus on these viruses has revealed the presence of several novel filoviruses in bats from Asia ${ }^{7,8,9,10}$, Africa $^{11,12,13}$ and Europe ${ }^{14,15}$. Currently, the only filovirus known to be endemic in Europe is Lloviu virus (LLOV), where genomic RNA was identified during the investigation of Schreiber's bats (Miniopterus schreibersii) die-off events in the Iberian-peninsula in $2002^{14}$. After its initial discovery, no other reports were published with regards to the presence of this virus until 2016, when RT-PCR positive bat carcasses in Hungary confirmed a more widespread presence of LLOV in M. schreibersii across Europe ${ }^{15}$. This was followed by a report on seropositivity among Schreiber's bats in Spain, suggesting the circulation of LLOV in that area ${ }^{16}$. The circumstances of the initial detection of LLOV in Hungary were similar to the original events in Spain, with multiple possibly related die-off events in the area, exclusively affecting Schreiber's bats ${ }^{15}$. Nearly two decades have now passed since the discovery of LLOV and many questions remain unresolved regarding the nature of the virus, the risk of zoonotic spillover, and most importantly, the pathogenic potential for bats and humans. Concerns regarding the possible pathogenicity of LLOV in humans remain high, since multiple studies have revealed its functional and genomic relatedness to $\mathrm{EBOV}^{17,18,19,20}$. Recently, a recombinant LLOV (rLLOV) rescue system was developed, in which the missing LLOV genome termini were complemented by homologous EBOV or MARV sequences. Although rLLOV is able to infect known target cells of EBOV, including human primary macrophages, the inflammatory response in human macrophages, a hallmark of Ebola virus disease, is not induced by rLLOV. This suggests that LLOV might be able to infect humans, 
but the infection might not lead to disease, similar to the non-pathogenic Reston virus ${ }^{21}$. Public health preparedness to filovirus zoonoses is highly related to the ecological attributes of wildlife hosts. Outbreaks in the human population are most likely initiated by spillover events from infected animals to humans ${ }^{22}$. In the case of MARV, these spillover events have been reported to correlate with the life cycle of the bat host, with increased zoonotic events occurring during the birthing period ${ }^{23,24}$. Importantly however, bat life cycles are different in temperate climate conditions than in the tropics. The annual life cycle of Schreiber's bats has key differences from bats that live in the tropics, most importantly the period of hibernation (Extended Figure 1).

In the past two decades, the identification of reservoir species of filoviruses has become a major focus of research, with numerous different vertebrate and arthropod taxa being studied $^{25,26,27}$. Interestingly, ectoparasites of bats have not been involved in many of these investigations, although they can carry several types of pathogens ${ }^{28}$. Bat flies are the most common ectoparasites of bats ${ }^{29}$. In the case of hippoboscoid flies, both sexes feed on the blood of the host species. Members of the Nycteribiidae and Streblidae families are typically associated with specific host species and in general bat flies and bats show a strong coevolution ${ }^{30}$. While bats are a major focus in viral emerging infectious disease research, the transmission patterns of these viruses within host bat populations and the role of their highly specialized ectoparasites in this enzootic ecology have barely been investigated to date ${ }^{28}$. Ectoparasites, however, represent perfect vector candidates for bat-to-bat transmission, including intraspecies and interspecies transmission ${ }^{31}$. It is therefore important to understand the complex picture of LLOV transmission within bats and potential vector species, including ectoparasites, to get a clearer picture about the natural circulation of this, and other, filoviruses.

In the present study, we sought to understand the biology of LLOV at a specific bat roost site in northeastern Hungary. We investigated the possible role of Schreiber's bats as natural reservoirs for LLOV and whether bat-associated parasites might play a role in the natural circulation of LLOV. We performed serologic and RT-PCR-based surveillance on Schreiber's bats from a site of previous LLOV detection in Hungary ${ }^{15}$ following a step-by-step investigation strategy. A primary result was the isolation of infectious virus directly from a bat blood sample, which makes LLOV only the second member of the family Filoviridae ever isolated from bats. 
Deployable field-sequencing technology based on target enrichment methods is not without precedent, as similar techniques were used extensively to sequence viruses from patient samples during the West African EBOV outbreak ${ }^{32,33}$. Here, we publish the use of such technology directly on wildlife hosts, optimized for the Nanopore sequencing platform that is amenable for both field and laboratory use in future studies.

In summary, we present evidence for asymptomatic LLOV infection of Schreiber's bats, seropositivity against LLOV, novel LLOV sequence data, and the presence of LLOV RNA in ectoparasites attached to LLOV-positive host bats. Isolation efforts provide the first infectious isolate of wild-type LLOV and also show the potential of the virus to infect bat, monkey, and multiple types of human cells in vitro.

\section{Results}

\section{LLOV seropositivity in Schreiber's bats}

Blood samples were collected from live bats and bat carcasses during the period of 2016-2019 at the site of LLOV detection in Northeastern Hungary in $2016^{15}$. In order to perform neutralization assays to detect the presence of anti-LLOV antibodies, we created lentivirus particles pseudotyped with the LLOV or EBOV glycoprotein (GP). Cross-reactivity with pseudotyped viruses (PVs) expressing GPs of other filoviruses was not tested due to the lack of sufficient amounts of bat sera. Importantly however, LLOV PVs did not cross react with EBOV convalescent serum (NIBSC WHO standard 15/262) (Extended Data Fig. 3). We used these PVs to perform antibody neutralization tests (PVNTs) on bat serum samples from both live and dead bats (Fig. 1, Table 1). 
A

Polyclonal antibodies or mABS
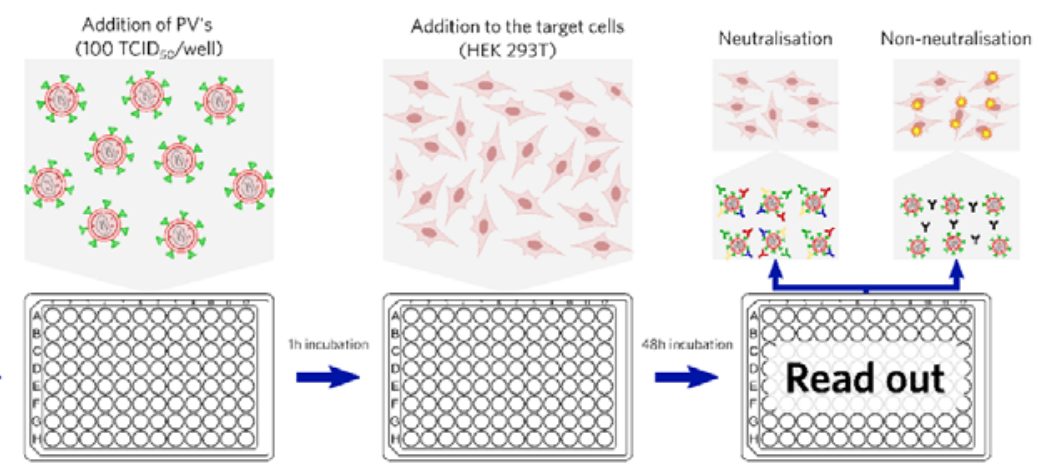

B
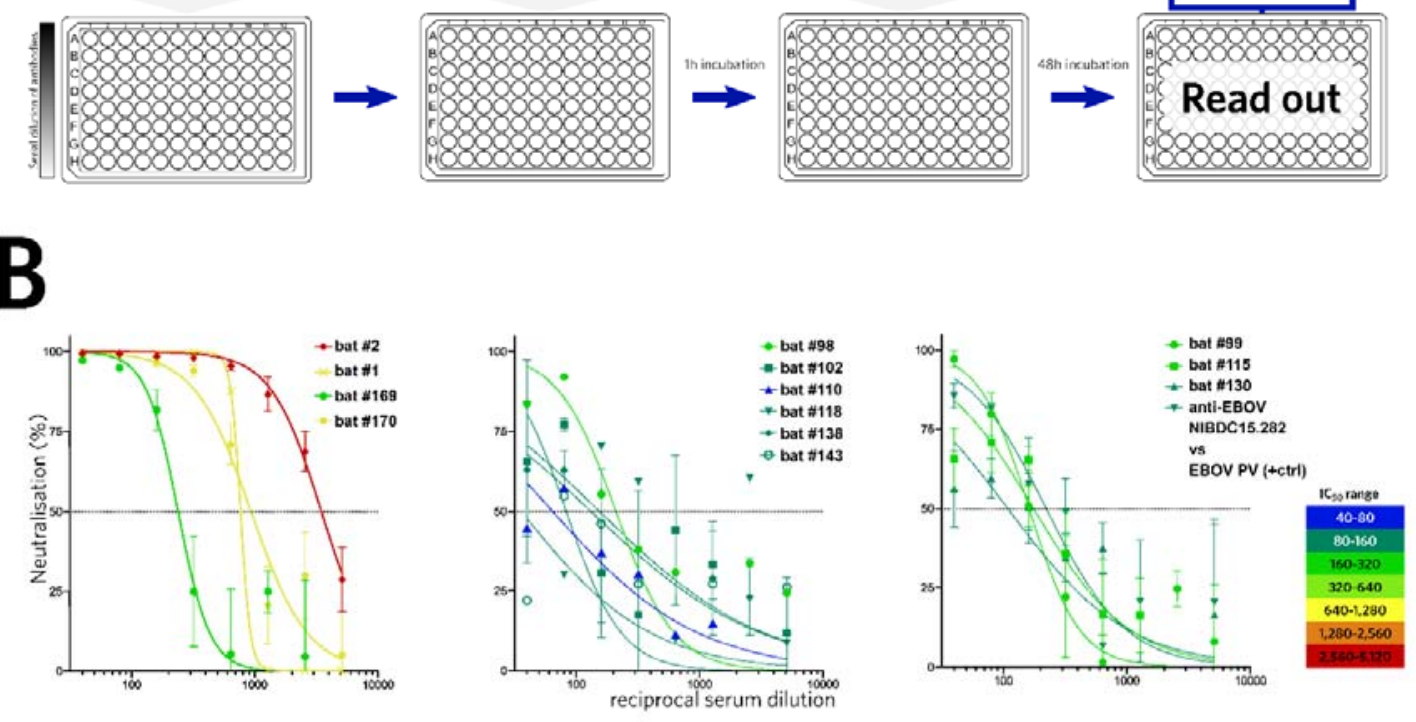

Fig. 1. - Pseudotyped virus neutralization test (PVNT) experiments of blood sera from Schreiber's bats.

Panel A - Workflow of PVNT; Panel B - PVNT results of LLOV seropositive bat sera. First graph represents dead animal samples (samples: bat \#2, bat \#1, bat \#169, bat \#170), whilst the second and third graph presents live bat samples (bat \#98 to bat \#130)

Four Schreiber's bat carcasses tested were seropositive for LLOV, with relatively high neutralization titres (254-3485 $\mathrm{IC}_{50}$ ). We were also able to detect LLOV RNA in certain tissues of these carcasses with low copy numbers, detailed in Table 1 and Table 2. We also investigated serum samples taken from 74 live Schreiber's bats from the first sampling event in September 2018. Seroprevalence among live sampled bats was 9/74 (12.16\%) with relatively weak to moderate titres $\left(64-211 \mathrm{IC}_{50}\right)$, particularly compared to seropositive carcases (Fig. 1, Table 1). Unfortunately, the highly limited amount of blood samples from live animals precluded replicate testing in certain cases, also hindering the possibility of coupled serologic and RT-PCR examination. Therefore we were able to conduct both serological and RT-PCR examination in only one case (LLOV_105) where LLOV RNA was detected along with seronegativity (Supplementary Table). 


\section{LLOV RNA in bats and related ectoparasites}

After the verification of seropositivity in bats, we focused on detection of LLOV RNA via RT-PCR in consecutive sampling events to facilitate viral genome sequencing and virus isolation efforts. Regular checking for dead animals during the hibernation period resulted in 10 bat carcasses (nine Miniopterus schreibersii, one Myotis myotis). After dissection of these carcasses, we tested blood and multiple organ samples (brain, liver, lung, spleen, and kidney) for the presence of LLOV and detected viral RNA in two (2/5) dead bats from 2016 and one from 2018 (Table 2). Overall, LLOV prevalence among Miniopterus carcasses was 33.3\% (3/9). Notably, LLOV RNA was only detected in spleen and lung samples, with low genomic copy numbers in both organs (Table 2).

Altogether, 779 samples ( 351 blood, 89 feces, 19 urine, 320 ectoparasites) were collected from live animals (351 - Miniopterus schreibersii, 2 - Myotis myotis) in seven sampling events and tested for the presence of LLOV RNA (Fig. 2A). We performed on-site RT-PCR analysis for the detection of viral RNA in blood samples from live animals, while other sample types were tested under laboratory conditions. On-site RT-PCR permitted the targeted examination and re-sampling of RT-PCR positive bats. Overall, 1.14\% (4/351) of livesampled animals were positive for LLOV by RT-PCR. Only the mid-to-late September sampling of bats in 2018 (LLOV_105, LLOV_147) and 2019 (LLOV_329, LLOV_378) provided positive blood samples, with a 4\% RT-PCR positivity rate in 2018 and $2.8 \%$ in 2019 (Fig. 2). We did not detect LLOV RNA positive animals in 2020, but only limited sampling was conducted due to COVID-19 pandemic-related restrictions. None of the RT-PCR positive animals showed signs of disease, and their general condition and activities appeared normal. 


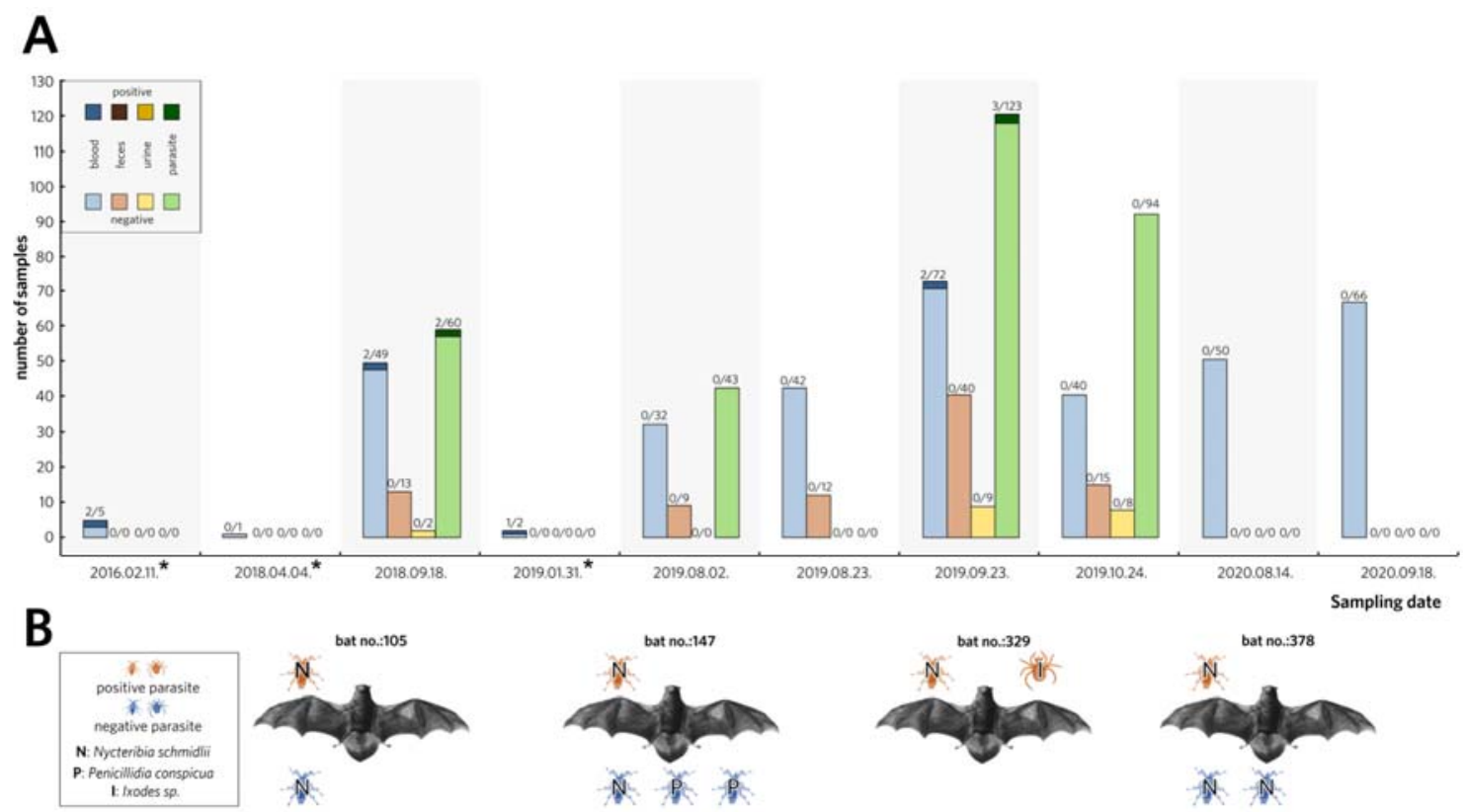

Fig. 2. - Summary of tested bat samples, verified RT-PCR positive bats and their ectoparasites. Panel A Number of LLOV-specific RT-PCR negative (lighter color) and positive (darker color) samples in each category: blood or organs (blue), feces (brown), urine (yellow), ectoparasites (green). *During these sampling events only passive monitoring was performed; Panel B - Schematic representation of coupled bat-ectoparasite LLOV RT-PCR positivity.

We collected feces samples from an RT-PCR positive bat (LLOV_378) to test the shedding characteristics of the virus and found no evidence of the presence of LLOV RNA in these samples (Fig. 2). All other feces $(n=89)$ and urine $(n=19)$ samples were retrieved from animals with RT-PCR negative blood and were found to be negative as well.

In order to get a clearer picture of possible bat-to-bat transmission pathways, we also examined the presence of LLOV RNA in bat-related ectoparasites, mostly from the family Nycteribiidae known as 'bat flies' along with some hard ticks (Ixodidae). In the process of collecting bat samples, specimens of bat flies including Nycteribia schmidlii $(\mathrm{n}=214)$, Nycteribia latreillii $(\mathrm{n}=2)$, Penicillidia conspicua $(\mathrm{n}=65)$ and Penicillidia dufouri $(\mathrm{n}=4)$, as well as Ixodes sp. $(\mathrm{n}=35)$ ticks were collected. Positive ectoparasites were only retrieved from positive bats, suggesting the presence of the virus exclusively in engorged parasites (Fig. 2, Supplementary Table). Altogether, we detected LLOV RNA in four Nycteribia schmidlii samples and one Ixodes sample from four RT-PCR positive bats (Fig. 2B). 
A detailed summary of all RT-PCR positive samples, including sample type/organ, $\mathrm{C}_{\mathrm{T}}$ values of RT-PCR reactions and indications of follow-up experiments are presented in Table 2.

\section{Lloviu virus isolation on bat, monkey and human cells}

Freshly obtained blood samples from the animals previously denoted PCR positive (LLOV_329, LLOV_378) were used for in vitro isolation experiments. Considering the small body size of these bats, the volume of these samples is routinely extremely small, usually between 5 and $20 \mu 1$. The initial in vitro isolation efforts, utilizing multiple cell lines (Vero E6 - African green monkey kidney and Tb1-Lu - bat lung cells) did not result in detectable LLOV replication. We neither observed any cytopathic effect in the cell culture experiments nor obtained positive real-time RT-PCR results after three blind passages.

After obtaining SuBK12-08 cells $^{34}$, we repeated these efforts using the LLOV_378 blood sample. After the second blind passage, we observed a strong cytopathic effect (Extended Data Fig. 5). Virus replication was further verified by real-time RT-PCR which resulted in lower real-time $\mathrm{Ct}$ value (18.469) than the original blood sample (30.1), clearly indicating an increase in viral genomic copy numbers. Viral titers of passage 4 virus were determined by tissue culture infectious dose $\left(\mathrm{TCID}_{50}\right)$ assay on SuBK12-08 cells and were as high as $1.78 \times 10^{9} \mathrm{TCID}_{50}$ units/ml. The established virus isolate was subjected for in vitro testing of other cell lines. Vero E6, SH-SY5Y (human neuroblastoma), HepG2 (human hepatocyte carcinoma), HCC78 (human lung adenocarcinoma), HCT116 (human colon carcinoma) were successfully infected with MOI 0.01 virus isolate. After ten days post-infection we measured a significant increase in viral genomic copy numbers. RT-PCR results of the cell supernatants are given (Extended Data Table 1).

\section{Sequencing of the viral genome}

We developed an ARTIC-like amplicon based (https://artic.network/) method for sequencing the complete coding region of the LLOV genome ${ }^{32,35,36}$. Using this method, we were able to retrieve sequence data from the RT-PCR positive bat from which virus was isolated (LLOV_378) and a nearly complete genome from its associated bat fly ectoparasite (LLOV_378_P1) (Table 2).

We generated sequence data for $\sim 99 \%$ of the known LLOV reference genome (NCBI accession NC_016144). In the case of sample LLOV_378, 99.45\% of the known genome was covered, starting from reference nucleotide position 28 and ending at 18,853. The mean 
coverage of this sample using all the data which was derived from the three different primer sets was 17,345× (Extended Data Fig. 2). The consensus sequence derived from the RT-PCR positive-associated parasite, sample LLOV_378_P1, spanned positions 28 to 18,853, with one notable gap between position 11,937 and 12,051. Importantly, there were no differences between the bat and bat fly derived viral sequences. Our novel LLOV sequence data are 99.198\% identical to the reference genome. We detected the highest level of sequence variation between the novel sequence data and the reference genome within the glycoprotein gene 2 (GP2) (Fig. 3). We also performed an indicative sequencing of the established infectious isolate. The obtained genomic information for this isolate is available under the accession number (NCBI accession: MZ541881). 


\section{A}

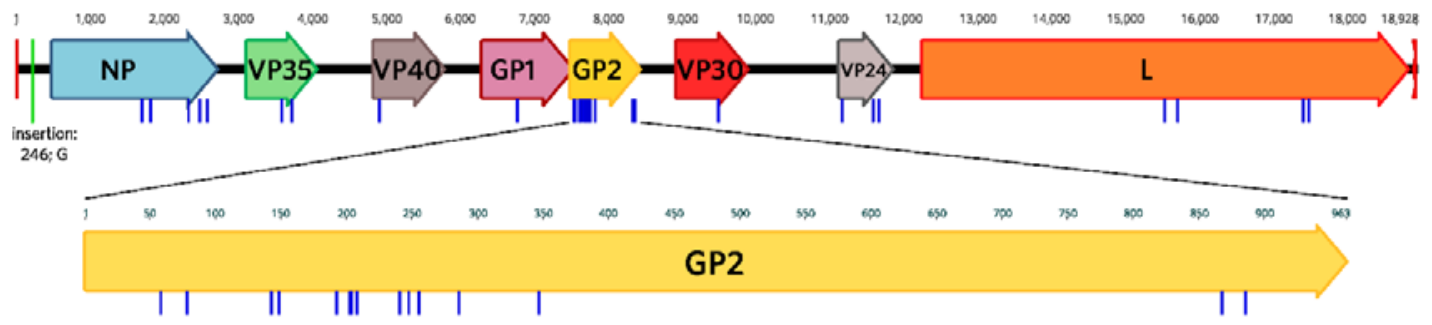

B

\begin{tabular}{|c|c|c|c|}
\hline Position (bp) & AA position in ORF & Transition & Gene \\
\hline 1,109 & 410 & $\mathrm{~N} \rightarrow \mathrm{T}$ & \multirow{5}{*}{$\mathrm{NP}$} \\
\hline 1,816 & 446 & $S \rightarrow P$ & \\
\hline 2.339 & 620 & $\mathrm{~N} \rightarrow \mathrm{S}$ & \\
\hline 2,485 & 669 & $\mathrm{~F} \rightarrow \mathrm{K}$ & \\
\hline 2,591 & 704 & $\mathrm{I} \rightarrow \mathrm{T}$ & \\
\hline 3,595 & 163 & $A \rightarrow T$ & \multirow{2}{*}{ VP35 } \\
\hline 3,730 & 208 & $\mathrm{~L} \rightarrow \mathrm{F}$ & \\
\hline 4,913 & 34 & $P \rightarrow L$ & VP4O \\
\hline 6.780 & 165 & $\mathrm{H} \rightarrow \mathrm{Y}$ & GP1 \\
\hline 7.536 & 20 & $\mathrm{~S} \rightarrow \mathrm{N}$ & \multirow{15}{*}{ GP2 } \\
\hline 7,556 & 27 & $E \rightarrow K$ & \\
\hline 7,620 & 48 & $L \rightarrow P$ & \\
\hline 7,626 & 50 & $L \rightarrow P$ & \\
\hline 7,670 & 65 & $S \rightarrow P$ & \\
\hline 7,680 & 68 & $V \rightarrow A$ & \\
\hline 7.681 & 68 & $V \rightarrow A$ & \\
\hline 7,685 & 70 & $\mathrm{Y} \rightarrow \mathrm{H}$ & \\
\hline 7,718 & 81 & $T \rightarrow P$ & \\
\hline 7,725 & 83 & $L \rightarrow P$ & \\
\hline 7,733 & 86 & $\mathrm{P} \rightarrow \mathrm{S}$ & \\
\hline 7.763 & 96 & $S, P$ & \\
\hline 7.824 & 116 & $V \rightarrow A$ & \\
\hline 8,345 & 290 & $\mathrm{~T} \rightarrow \mathrm{A}$ & \\
\hline 8,363 & 296 & $\mathrm{I} \rightarrow \mathrm{V}$ & \\
\hline 9,498 & 195 & $A \rightarrow T$ & VP30 \\
\hline 11,159 & 16 & $\mathrm{~F} \rightarrow \mathrm{L}$ & \multirow{3}{*}{ VP24 } \\
\hline 11.585 & 158 & $\mathrm{D} \rightarrow \mathrm{N}$ & \\
\hline 11.667 & 185 & $C \rightarrow Y$ & \\
\hline 15,522 & 1,098 & $G \rightarrow R$ & \multirow{5}{*}{ L } \\
\hline 15,697 & 1,156 & $D \rightarrow G$ & \\
\hline 17,389 & 1,720 & $S \rightarrow L$ & \\
\hline 17,401 & 1,724 & $R \rightarrow Q$ & \\
\hline 17,469 & 1,747 & $\mathrm{~T} \rightarrow \mathrm{S}$ & \\
\hline
\end{tabular}
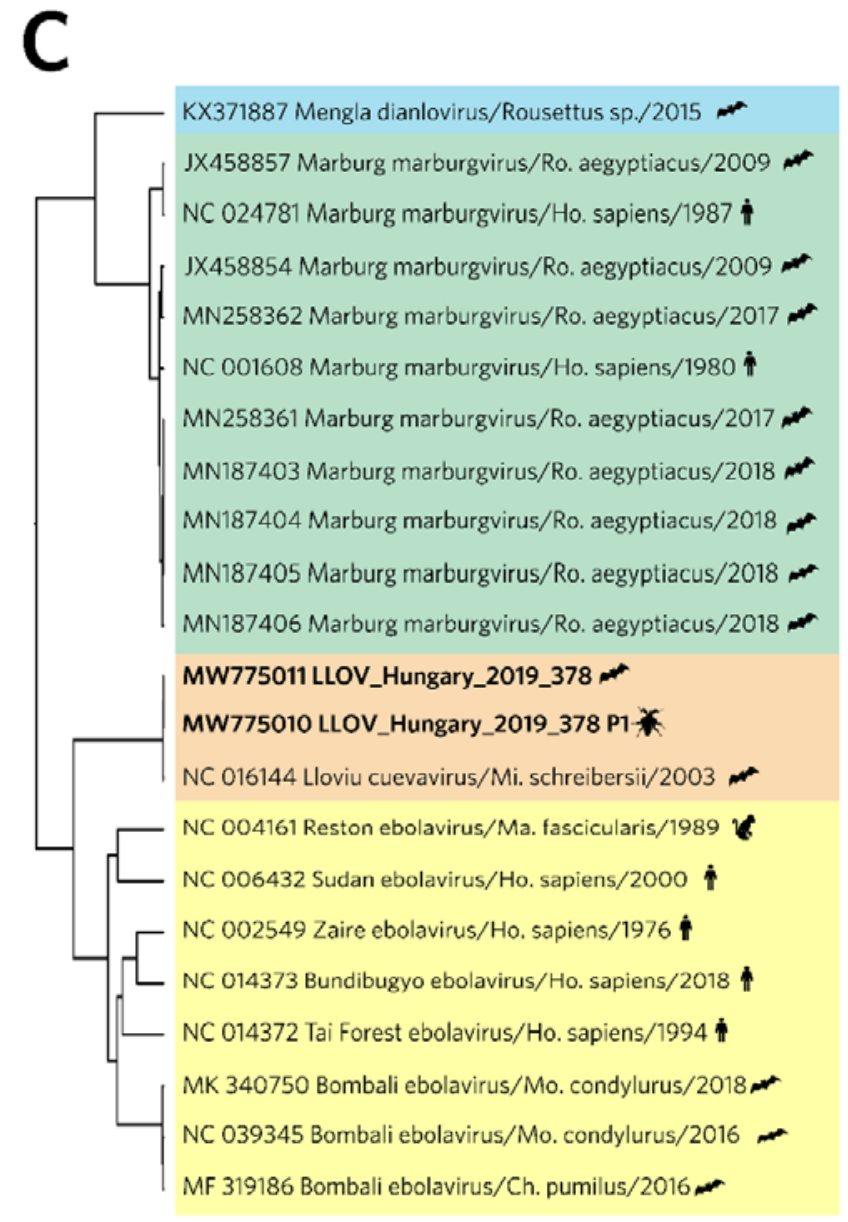

Fig. 3. Genomic and phylogenetic attributes of the Lloviu virus genome sequences presented in this manuscript. Panel A: Annotated Lloviu virus genome sequence from bat derived sample LLOV_378 (Genbank MW775011). Blue bars indicate differences in amino acid sequences and the green bar represents an insertion compared to the Spanish LLOV reference isolate (GenBank: NC_016144); Panel B: Summary table of amino acid coding differences between LLOV_378 and NC_016144; Panel C: Phylogenetic analysis of select complete genome sequences representing four filovirus genera including LLOV_378 (Genbank: MW775011) and LLOV_378P1 (Genbank: MW775010). Sequences are colored by genus Dianlovirus (blue), Marburgvirus (green), Cuevavirus (orange), and Ebolavirus (yellow)). Host Taxon name abbreviations: Ro. - Rousettus; Ho. Homo; Mi. - Miniopterus; Ma. - Macaca; Mo. - Mops; Ch. - Chaerephon 


\section{Discussion}

Several novel members of the family Filoviridae have been discovered in the last decade including some found in Europe and Asia, raising the potential for the emergence of filoviruses outside of Africa. There is a considerable knowledge gap regarding the pathogenicity, animal hosts, and transmissibility of these newly discovered viruses.

The most exciting result presented here is the successful isolation of infectious LLOV from the blood sample of an asymptomatic but RT-PCR positive bat. The pronounced cytopathic effect of LLOV-infected Miniopterus SuBK12-08 cells combined with the high titers of virus stocks propagated in these cells suggest that SuBK12-08 cells are highly permissive to LLOV infection. This, along with our other results, provides strong indication for the reservoir role of Schreiber's bats and opens the possibility for extended pathogenicity studies in the near future. As we presented here, Lloviu virus has the potential to infect human cells, therefore receptor identification, gene expression and most importantly antiviral studies are urgently needed.

Here, we describe the circulation of LLOV in a colony of Schreiber's bats over the span of several years. This study represents the first detailed observation of the natural circulation of a filovirus in a temperate climate region.

The seropositivity and presence of LLOV RNA in these bats and the observation of asymptomatic animals are supportive of a putative role of Schreiber' $s$ bats as the natural reservoir hosts for this virus ${ }^{37}$. Isolation of the infectious virus directly from the blood sample of an asymptomatic animal gives strong evidence for the reservoir role. This is in line with the previous finding that Egyptian fruit bats ( $R$. aegyptiacus) are a reservoir for marburgviruses ${ }^{38,39}$, and other bat species have been suggested as possible reservoirs for other filoviruses, such as the Bombali virus ${ }^{11}$. We detected LLOV RNA in multiple deceased bats, raising the possibility that these bats died as a consequence of infection. Interestingly, some individuals were found with respiratory tract bleeding, however there was no correlation to RT-PCR positivity (Supplementary Table). More sophisticated surveillance activities are necessary to better understand the nature of LLOV infection in bats and to clarify the possible role of the virus in the morbidity and mortality among these bats. Although rare, the occurrence of both fatal and non-fatal outcomes of viral infections of bats is not unique. The best known pathogens to cause such patterns in bats are lyssaviruses ${ }^{40}$. 
We also developed a sensitive serological test based on pseudotype virus neutralization that supports LLOV serosurveillance testing of bat sera samples. Before this study, only immunoblot analysis had been used for LLOV serologic examination in bats ${ }^{16}$. Our method enabled the serological study of live-sampled wild bats, showing a LLOV seroprevalence of $12.2 \%(9 / 74)$, which is lower than the previously published $36.5 \%$ seroprevalence from bats in Spain, though our test measures antibody neutralization rather than simply binding activity. This is also direct evidence of LLOV exposure in these bats, which supports the previous observation of a more extended spatio-temporal presence of the virus in Europe ${ }^{15}$.

Except for MARV in Egyptian fruit bats, there is little information about enzootic circulation of a filovirus among specific bat colony sites. MARV virus prevalence values seen in various countries range from $2.5 \%$ and $13.7 \%$, with seasonal variability noted ${ }^{6,41,23}$. Based on previous studies, natural fluctuation of the age composition within a single colony and therefore the changing numbers of susceptible animals within the population may largely affect the prevalence ${ }^{23}$. In case of Pteropodidae (fruit bats), bi-annual birth pulses may facilitate the persistence of filoviruses ${ }^{24}$. Available data on MARV circulation highlighted juvenile bats (around $\sim 6$ months old) as particularly likely to be infected at specific times of the year. This seasonal emergence of juvenile Egyptian fruit bats coincides with increased risk of human infection ${ }^{23}$. In contrast to some tropical species, $M$. schreibersii bats have only one breeding season per year, and notably have a hibernation period. Considering these features, there should be other driving factors involved in LLOV circulation (Extended Data Fig. 1)

We have also shown the first evidence of filovirus RNA presence in arthropods. However, the precise role of bat flies and other ectoparasites in LLOV transmission is unknown. It is not yet clear whether these bat flies may be acting as natural vectors or mechanical vectors for LLOV transmission or are simply dead-end LLOV spillover hosts. LLOV-positive ectoparasites were only retrieved from positive animals, suggesting the presence of the viral RNA in engorged parasites. Coupled sequencing results from host and parasite also support passive transmission without active viral replication, since no differences were found in the viral genomic sequences derived from the bat fly (LLOV_378_P1) and its bat host (LLOV_378). Given the current relatively limited dataset, it is not clear whether these ectoparasites play a role as a vector in the transmission of LLOV. However, we cannot rule out the possibility that these parasites serve as mechanical vectors in the transmission of LLOV, which warrants further studies. Compared to previous studies, Reston virus was not found to replicate in arthropod 
vectors and MARV RNA was not found in parasites on MARV-positive bats ${ }^{42,43,44}$. Based on the successful sequencing of LLOV RNA isolated from bat flies, resulting in high-quality sequence data, indirect LLOV surveillance could potentially be conducted by testing ectoparasites of $M$. schreibersii bats, obviating the need for invasive blood sampling of bats. This would provide the opportunity to conduct wide scale geographic surveillance to discover LLOV endemic regions and obtain a more detailed distribution map of LLOV infection among M. schreibersii populations. The reliability of ectoparasite-based surveillance strategy of bats was already reported for multiple bacterial species ${ }^{45}$.

Based on our novel sequence data, we have shown the first insights into the natural genomic evolution of LLOV. Comparing the LLOV genomic sequence data from this study with the reference genome sequence from Spain, the level of LLOV sequence variation is consistent with other filovirus genera. For ebolaviruses and marburgviruses, the GP gene is a hotspot of nucleotide sequence variation ${ }^{46,47}$. The consistency of elevated GP sequence variation compared to other genomic regions in all three genera (Ebolavirus, Marburgvirus, and Cuevavirus) suggests similar genetic evolutionary driving forces on this region (e.g. immune evasion).

\section{Conclusions}

Based on the presented data and considering the previous reports from $\operatorname{Spain}^{14,16}$, there are multiple reports of LLOV infection in Schreiber's bats, consistent with the idea that this species is a natural reservoir of the virus. Despite many remaining questions, we provide evidence and further support for the role of bats in the natural circulation of filoviruses in general. The transmission cycle of LLOV may be complex and potentially different from filoviruses whose enzootic cycle occurs in tropical climates. As a major difference, we present data showing LLOV RNA-positive highly-specialized bat-associated ectoparasites, highlighting the importance of future research regarding the role of these insects in LLOV and other filovirus transmissions. Similar surveillance studies may greatly facilitate the discovery and understanding of novel filoviruses. Most importantly, we isolated the infectious virus which represents the first isolate of a filovirus that does not belong to the ebola- or marburgvirus genera, and it is the second successful isolation of a filovirus directly from bats, following MARV, opening the door to further virological studies. Importantly, we show that multiple human cell lines are permissive to LLOV infection, which raises concerns about 
potential bat to human spillover events with LLOV in Europe and urges pathogenicity and antiviral studies.

\section{Materials and Methods (3000 words)}

\section{Ethics and biosafety statement}

Bat sampling activities on a country-wide scale were approved by the Hungarian Government Office of Pest County under the registration number of PE-KTFO/4384-24/2018. Animal handling was performed by licensed chiropterologists, no animals were harmed during the study, and all ethical standards were followed during the work. All in vitro virus isolation procedures were performed under Biosafety Level 4 conditions within the laboratory of Szentágothai Research Centre, University of Pécs, Hungary.

\section{Animal sampling}

We conducted regular bat colony monitoring during the hibernation period (between November and March) (passive surveillance) at the site of LLOV re-emergence in Hungary (Northeast Hungary, Zemplén Mountains) from 2016 to 2020, collecting only deceased bats, if found. Whole carcasses were frozen and transferred in a dry shipper to the biosafety level 4 (BSL-4) laboratory at the Szentágothai Research Centre, Pécs, Hungary, where they were dissected. Lung, spleen, kidney, liver, and brain tissues as well as blood samples were stored at $-80^{\circ} \mathrm{C}$ until further analyses.

In addition, seven live animal sampling events (active surveillance) were performed between 2018 and 2020, when samples (blood, urine, feces, ectoparasites) were collected from Schreiber's bats (Miniopterus schreibersii) and Greater mouse-eared bats (Myotis myotis). Bat species identification was performed by trained chiropterologists according to morphological identification keys $^{48}$. Considering conservational aspects, all sampling activities were conducted after the reproduction period of the colony (between August to November). During the live sampling events, serum samples were taken from captured bats after which they were left hanging separately in disposable paper bags (air permeable) for approximately $2 \square$ hours, while on-site RT-PCR analysis for the presence of LLOV RNA in from blood samples was performed (as detailed in the Virus detection section). This methodology permitted the observation and re-sampling of infected bats. Altogether, 376 bat individuals were sampled between 2016 and 2020 (Supplementary Table). Whole blood (maximum of $50 \mu \mathrm{L}$ ) was taken from the uropatagium vein from each animal by Minivette ${ }^{\circledR}$ POCT (Sarstedt, Germany) 
disposable microtubes. At the first live animal sampling event (18.09.2018), the blood samples were collected in 1.5-ml Eppendorf tubes, where serum was separated for neutralization assays by low-speed centrifugation $(3,000 \mathrm{rpm})$ for five minutes. Cell pellets were used for nucleic acid extraction and RT-PCR detection of LLOV RNA. Due to the multipurpose nature of the investigation and the strong limitation of the blood amount, in case of samples less than $8 \mu \mathrm{L}$, only LLOV RNA detection was conducted. When more blood was collected (approximately 8-13 $\mu \mathrm{L}$ ), only serology was performed. For samples with volumes above $13 \mu \mathrm{L}$, both LLOV RT-PCR and serology testing were performed.

Following the first live sampling, we changed the blood sampling methodology in order to provide the possibility of in vitro isolation efforts. During the following six live sampling events (02.08.2019-18.09.2020), freshly obtained blood samples were transferred directly to $200 \mu \mathrm{L}$ Virus Transport Medium (VTM) (UniTranz-RT $1 \mathrm{~mL}$ Transport System, Puritan, USA), and mixed gently by pipetting. A $100 \mu \mathrm{L}$ portion of each sample was deposited for nucleic acid extraction while the remainder was stored and served as inoculum for virus isolation experiments. When possible, urine, feces, and ectoparasites (ticks, bat flies) were also collected with forceps during bat handling. Feces and urine samples and ectoparasite specimens were immediately individually frozen in liquid nitrogen, and stored at $-80^{\circ} \mathrm{C}$ until further laboratory processes. Ectoparasites were identified based on morphological characteristics, identifying parasite species or genus according to generally approved reference keys using a stereomicroscope ${ }^{49,50}$.

\section{Virus detection}

For LLOV RNA detection, we performed RT-PCR tests from all sample types (lung, spleen, kidney, liver, brain tissues and blood from carcasses; blood, urine, feces samples and ectoparasite specimens from live bats). For blood samples in VTM, we carried out on-site LLOV RNA detection in the field, while other samples were transported to the laboratory for testing.

Tissues, feces, and ectoparasites were homogenized in $100 \mu \mathrm{L}$ 1x Phosphate Buffered Saline (PBS) using Minilys Personal Homogenizer (Bertin, Germany) with glass beads. The urine samples were complemented with $1 \mathrm{x}$ PBS to a total volume of $100 \mu \mathrm{L}$. Then, the samples were centrifuged at $10,000 \mathrm{rpm}$ for 5 minutes and $100 \mu \mathrm{L}$ supernatant was transferred for RNA extraction. The $100 \mu \mathrm{L}$ blood samples in VTM were directly used for RNA extraction. 
RNA was extracted using Direct-Zol RNA MiniPrep (Zymo Research, USA). RNA was then used for RT-PCR using the QIAGEN OneStep RT-PCR kit (Qiagen, Germany) at $50^{\circ} \mathrm{C}$ for 30 minutes, and $95^{\circ} \mathrm{C}$ for 15 minutes, followed by 45 cycles of $95^{\circ} \mathrm{C}$ for 15 seconds, $60^{\circ} \mathrm{C}$ for 20 seconds $72^{\circ} \mathrm{C}$ for 40 seconds (the fluorescence signal was detected during the annealing step). We used the primers FiloAneo: 5'-ARG CMT TYC CAN GYA AYA TGA TGG T-3' and FiloBNeo: 5'-RTG WGG NGG RYT RTA AWA RTC ACT NAC ATG-3' and the probe Lloviu-S: FAM-5'-CCT AGA TTG CCC TGT TCA TGA TGC CA-BHQ1-3 ${ }^{\prime 16}$. It is noteworthy that a previously described detection method published by our laboratory ${ }^{15}$ resulted in a significant number of false positives, which in certain cases we subsequently failed to validate with a pan-filovirus nested-PCR method and sequencing ${ }^{7}$. All experiments were run on the MyGo Pro PCR system platform (IT-IS Life Science, Ireland).

Virus isolation

Cell lines used in this study: Cercopithecus aethiops (African green monkey kidney ) cells (Vero E6; ATCC ${ }^{\circledR}$ CRL-1586 $^{\text {TM }}$ ), Miniopterus sp. kidney cells (SuBK12-08; kindly provided by Ayato Takada, Hokkaido University) ${ }^{34,18}$, human neuroblastoma cells (SH-SY5Y; ATCC ${ }^{\circledR}$ CRL-2266 ${ }^{\mathrm{TM}}$ ), human hepatocellular carcinoma (Hep G2; ATCC HB-8065 ${ }^{\mathrm{TM}}$ ), human nonsmall cell lung carcinoma (HCC-78; DSMZ ACC 563), human colon carcinoma (HCT 116; DSMZ ACC 581), Tadarida brasiliensis lung cells (Tb $1 \mathrm{Lu}$; ATCC ${ }^{\circledR} \mathrm{CCL}^{-88^{\mathrm{TM}}}$ ).

Vero E6 cells were maintained in Dulbecco's modified Eagle medium (DMEM, Lonza Cat.No: 12-604F.), SH-SY5Y and Hep G2 cells were maintained in DMEM with $1 \%$ of MEM Non-essential Amino Acid Solution 100x (NEAA) (Sigma Cat. No: M7145), SuBK1208 and $\mathrm{Tb} 1 \mathrm{Lu}$ in Eagle's Minimum Essential Medium (EMEM, Lonza Cat. No: 12-662F) with $1 \%$ of L-Glutamine (200 mM) (Lonza Cat. No: BE17-605E), HCC-78 in RPMI-1640 (Sigma Cat. No: R5886), HCT 116 in McCoy's 5A (Lonza, Cat. No: BE12-688F). All culture medium was supplemented with $10 \%$ heat inactivated Fetal Bovine Serum (FBS) (Gibco Cat.No: 16140071), and 1\% Penicillin/Streptomycin (10,000 U/mL). All cell lines were grown at $37^{\circ} \mathrm{C}, 5 \% \mathrm{CO}_{2}$.

Freshly obtained blood samples from field PCR-tested LLOV RNA-positive bats were frozen in liquid nitrogen and transferred to the BSL-4 laboratory at the Szentágothai Research Centre to perform virus isolation experiments. Of note, due to the low body weight of the animals, the blood sample volumes are extremely low, usually below $50 \mu \mathrm{L}$. The blood samples (5-50 
$\mu \mathrm{L}$, depending on the original amount) were complemented with cell culture media to a volume of up to $50 \mu \mathrm{L}$ and used to inoculate cell monolayers seeded in 24-well plates with $\sim 80 \%$ confluency. After an 1-hour incubation at $37^{\circ} \mathrm{C}$ and $5 \% \mathrm{CO}_{2}$, the cells were washed once with cell culture media. The cells were monitored daily for cytopathic effects. After 7 days, we performed two freeze-thaw cycles before culture supernatants were transferred to fresh cells. After three blind passages, cells were lysed by the freeze-thaw method, subjected to nucleic acid extraction by Direct-Zol RNA MiniPrep (Zymo Research, USA) and analyzed for the presence of LLOV RNA by RT-PCR using LLOV-specific primers. Viral titers were determined by tissue culture infectious dose $\left(\mathrm{TCID}_{50}\right)$ assays on SuBK12-08 cells using the Spearman-Karber algorithm.

\section{Generation of LLOV pseudotyped lentiviruses and neutralization tests}

The LLOV GP gene (GenBank accession JF828358) was inserted into the pCAGGS expression plasmid (kind gift of Prof Ayato Takada). Generation of LLOV and EBOV GP pseudotyped lentiviruses was based on a protocol described previously ${ }^{51}$, with co-transfection of three plasmids, pCAGGS-LLOV GP or pCAGGS-EBOV GP, p8.91 (HIV-1 gag-pol) and pCSFLW (luciferase reporter), into HEK293/17 cells, using $1 \mathrm{mg} / \mathrm{mL}$ branched Polyethyleneimine (PEI) (Sigma-Aldrich, USA) at a ratio of 1:10 ( $\mu \mathrm{g}$ DNA: $\mu \mathrm{L}$ PEI). Pseudotyped lentivirus (PV) supernatant was harvested $48 \mathrm{~h}$ later and stored at $-80^{\circ} \mathrm{C}$. Titration of PVs was carried out as described elsewhere ${ }^{52}$ by 2 -fold serial dilution across white Nunc flat-bottomed 96-well microplates (ThermoFisher Scientific, USA) in HEK293T/17 target cells $\left(2 \times 10^{4} /\right.$ well), incubated at $37^{\circ} \mathrm{C}, 5 \% \mathrm{CO}_{2}$. After $48 \mathrm{~h}$, the media was removed and discarded; Bright-Glo reagent was added to the plate and incubated at room temperature for $5 \mathrm{~min}$ before measuring luminescence on a GloMax 96 luminometer (Promega, USA), with titers given in Relative Light Units (RLU) per mL. For ease of interlab comparison, $\mathrm{TCID}_{50}$ titers were also obtained using the same methodology but using a 5fold dilution series.

Luminescence values were used to calculate the PV titre $\left(\mathrm{TCID}_{50} / \mathrm{mL}\right)$ using the ReedMuench method ${ }^{53}$. The cumulative number of positive and negative wells for PV infection at each dilution was determined and the percentage calculated for each. The threshold value for a positive well was set at $2.5 \mathrm{x}$ the average luminescence value of the cell-only negative controls (Extended Data Fig. 2). 
Pseudotyped virus neutralization tests (PVNTs) were performed in white microplates by serial dilution of bat sera $(1: 40$ to 5,$120 ; 1: 100$ to 12,800 or $1: 200$ to 25,600$)$ with PVs $\left(\sim 1 \times 10^{5}\right.$ $\mathrm{RLU} /$ well or $\sim 100 \mathrm{TCID}_{50} /$ well, calculated according to the titration result) incubated at $37^{\circ} \mathrm{C}$ for $1 \mathrm{~h}$. Target HEK293/17 cells were then added and plates read as described previously ${ }^{54,52}$. In total, 78 serum samples, mainly from live bats, were tested. Control serum standards (WHO NIBSC15/262 \& NIBSC15/282 antibody standards) obtained from EBOV disease convalescent patients were used, as no LLOV positive serum samples were available. Data was normalized to \% reduction in luminescence with respect to the average RLU of cell only (100\% neutralization) and PV only (0\% neutralization) controls and fitted into a non-linear regression model (log [inhibitor] vs. normalized response, variable slope). $\mathrm{IC}_{50}$ antibody titres were calculated using Prism 8 software. Average values of two independent experiments are indicated unless otherwise stated. The cut-off for PVNT positivity was determined using $\mathrm{IC}_{50}$ values from samples where the total antibody-mediated reduction in RLU was less than $20 \%$ of the PV + no serum control (i.e. $0 \%$ neutralization) or when a neutralization of $<70 \%$ was achieved. The mean of these values was calculated and a cut-off was defined for values higher than the mean + three times the standard deviations ${ }^{55,56,57}$ (Extended Data Fig. 3).

\section{Amplicon-based Nanopore sequencing}

We developed an amplicon sequencing method based on previous protocols ${ }^{58,59}$ that is able to amplify the LLOV specific gene regions in two parallel multiplex PCR reactions ${ }^{36}$. LLOV RNA positive samples LLOV_378 and LLOV_378P1 were used for sequencing. cDNA preparation from RNA samples was conducted with SuperScript IV (Invitrogen, USA) with random hexamers. Amplicons were generated from cDNA with Q5 Hot Start HF Polymerase (New England Biolabs, USA), with three different primer sets in parallel pools (namely: LLOV_400bp pool 1 and 2, LLOV_500bp pool 1 and 2, LLOV_2000bp pool 1 and 2) (Extended Data Fig. 2). $2.5 \mu \mathrm{L}$ amplicons from each pool belonging to the same primer set (Primer set: 400 or 500 or 2000) were diluted in $45 \mu \mathrm{L}$ nuclease-free water before the end repair and dA tailing were performed with the NEBNext Ultra II End Repair/dA-Tailing Module (New England Biolabs, USA). $1.5 \mu \mathrm{L}$ end-prepped DNA was transferred to the next reaction directly and the barcodes derived from EXP-NBD196 (Oxford Nanopore Technologies, UK) were ligated with NEBNext Ultra II Ligation Module (New England Biolabs, USA). After the clean up of the pooled different barcoded samples jointly with Ampure XP beads (Beckman Coulter, USA), the AMII sequencing adapters were ligated with 
NEBNext Quick Ligation Module. The final library was quantified with Qubit dsDNA HS Assay Kit (Invitrogen, USA) on a Qubit 3 fluorometer. $25 \mathrm{ng}$ final libraries were loaded onto a R9.4.1 (FLO-MIN106D) flow cell and were sequenced for 48 hours $^{36}$.

\section{Bioinformatic pipeline}

The raw sequencing data were basecalled using guppy (ONT guppy v4.4.2.) high accuracy basecaller algorithm (dna_r9.4.1_450bps_hac config file). Demultiplexing and trimming of barcodes were performed also with guppy using default parameters of 'guppy_barcoder' runcode. The ONT guppy software was used under Ubuntu Linux 18.04. Because ARTIC-like protocols generate chimeric reads, we performed length filtering with appropriate lengths specific to the used primer set (LLOV_400bp: 350-600 bp, LLOV_500bp: 440-700 bp, LLOV_2000bp: 1,400-2,700 bp). Primers were trimmed with the BBDuk (v38.84) Geneious Prime (v2021.1.1) plugin. To generate a consensus sequence, the processed reads from all samples were mapped to the LLOV reference genome (NCBI accession number: NC_016144) with the usage of MiniMap $2.17^{60}$. The generated consensus sequences were manually checked for basecalling errors.

\section{Sequencing of the virus isolate}

Prior to Nanopore sequencing, virus isolates were exposed to enrichment protocol as detailed previously ${ }^{61,62}$. Thereafter, nucleic acid was extracted from samples using the Direct-zol RNA Miniprep Plus Kit (Zymo Research, USA). Samples were then subjected to the Sequence Independent Single Primer Amplification (SISPA) approach with minor modifications to the previously described protocol ${ }^{62}$. cDNA amplification was performed by SuperScript IV Reverse Transcriptase (ThermoFisher scientific) with dNTPs $(10 \mu \mathrm{M})$ and $2 \mu \mathrm{M}$ K-8N primer following the manufacturer's instructions. In order to convert double stranded cDNA from first strand cDNA, samples were subjected to Klenow reaction. Thereafter, the products underwent a purification step using Agencourt AMPure XP beads (Beckman Coulter). Following that, ds cDNA was amplified by Q5® High-Fidelity DNA Polymerase (NEB). Amplified cDNA was purified by Agencourt AMPure XP beads (Beckman Coulter) and quantified using a Qubit dsDNA BR Assay kit (Thermo Fisher Scientific). The double stranded fragments were end prepped with NEBNext Ultra II End Repair/dA-Tailing Module (New England Biolabs, USA) and were barcoded with EXP-NBD196 (Oxford Nanopore 
Technologies, UK) kit. The final library was sequenced on a R9.4.1 (FLO-MIN106D) flow cell.

The raw sequencing data were basecalled using guppy (ONT guppy v5.0.7.) super-accuracy basecaller algorithm (dna_r9.4.1_450bps_sup config file). Demultiplexing and trimming of barcodes were performed also with guppy using default parameters of 'guppy_barcoder' runcode. The ONT guppy software was used under Ubuntu Linux 18.04. Porechop v0.2.4 was used to trim primer sequences used for SISPA amplification. To generate a consensus sequence, Medaka v1.4.2 was used to map the trimmed reads against the LLOV reference genome (NCBI accession number: NC_016144) and perform variant calling. Extension of the genome ends was done by manually filling in the observed overhangs based on a mapping performed using the Long Read Support tool in CLC Genomics workbench v20.0.4. Extension was stopped when read coverage fell below 20x.

\section{Phylogenetic tree caption:}

Prior to the phylogenetic tree implementation, 22 full length sequences belonging to the family Filoviridae were aligned in the MAFFT webserver (version 7 https://mafft.cbrc.jp/alignment/server/) using default parameters. Subsequently, a best substitution model selection was performed in IQ-tree webserver (http://iqtree.cibiv.univie.ac.at/) and thus a Bayesian phylogeny tree was generated using BEAST v1.10.4 ${ }^{63}$ under a GTR+G+I substitution model, assuming a constant population size and a strict molecular clock (uniform rates across branches). The MCMC chains were run for $10,000,000$ iterations and sampled each 1,000th generation. The effective sampling size was checked in Tracer software (>200). Thereafter, the Maximum clade credibility tree (MCC) was generated using TreeAnnotator and edited in the iTol webserver (https://itol.embl.de/). 


\section{Extended Data}

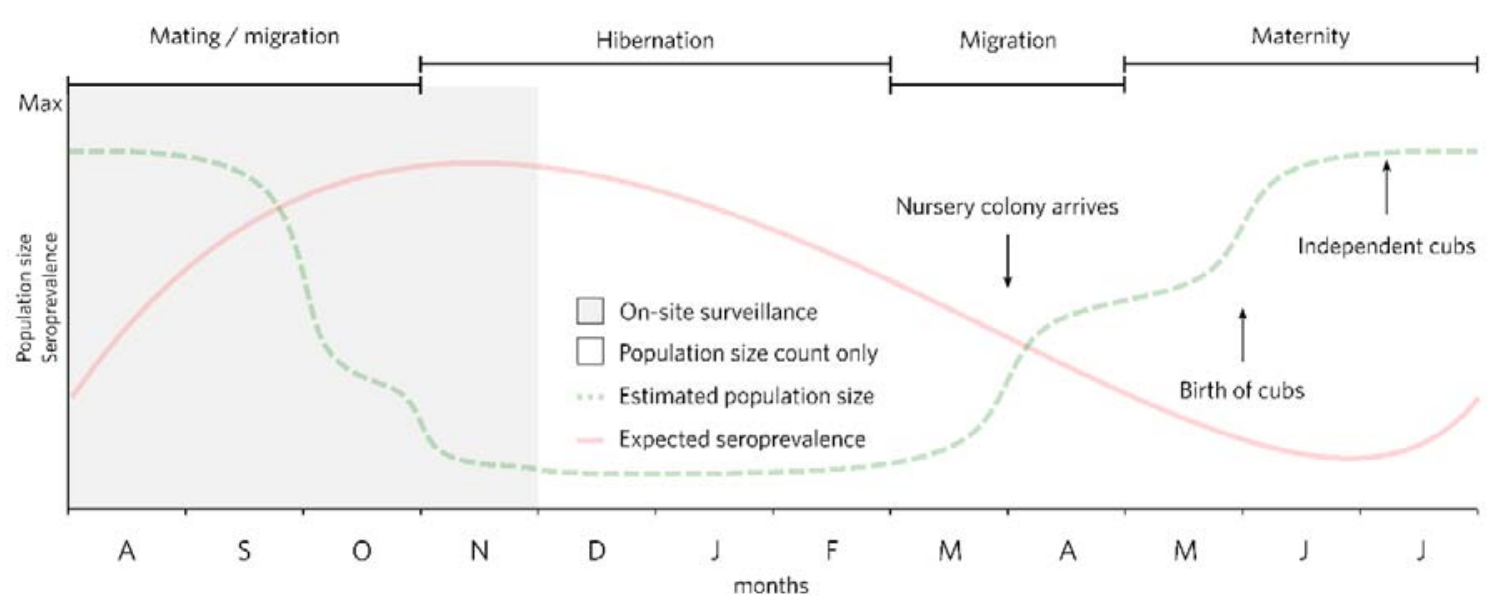

Extended Data Fig. 1. Annual cycle of the Schreiber's bats colony at the investigated mine in Northeast

Hungary. During hibernation, regular observation was performed of the bat colony with no active sampling.

Grey background represents the timeframe of active bat sampling and sample collection. Population size fluctuation is based on colony size monitoring data in recent years.

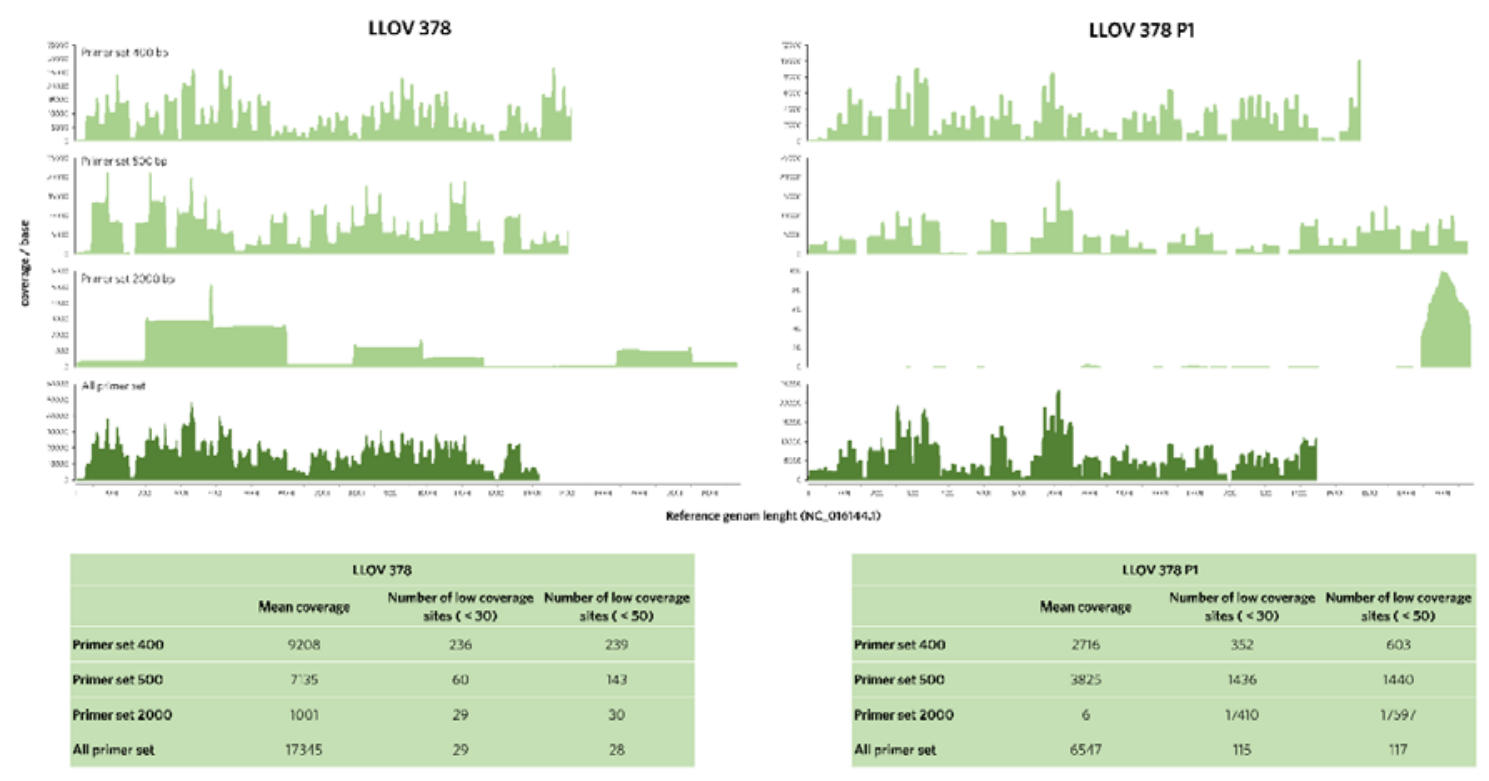

Extended Data Fig. 2. Coverage plot of sequenced samples LLOV_378 and LLOV:378_P1 alongside the reference LLOV genome sequence (NC_016144). Main characteristics of coverage (mean, extent) with the three different primer sets are shown in the tables below the plots. 

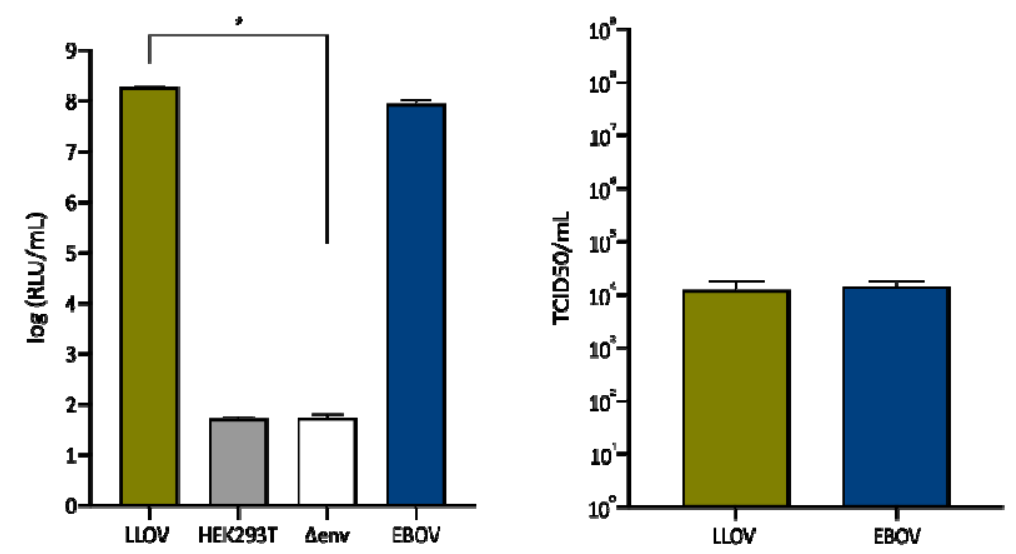

Extended Data Fig. 3. Lentiviral LLOV PV titres. An EBOV GP PV generated and titrated previously was included as a positive control in - infectivity and Panel B - TCID 50 assays. Uninfected cells (HEK293T) and particles devoid of GP ( $\triangle \mathrm{env})$ were included as negative controls. Average titre of at least three independent experiments shown. The error bars indicate the standard deviation. Statistical significance $(* \mathrm{p}<0.05$ MannWhitney test) in comparison to $\Delta$ env determined with Prism 8 software.

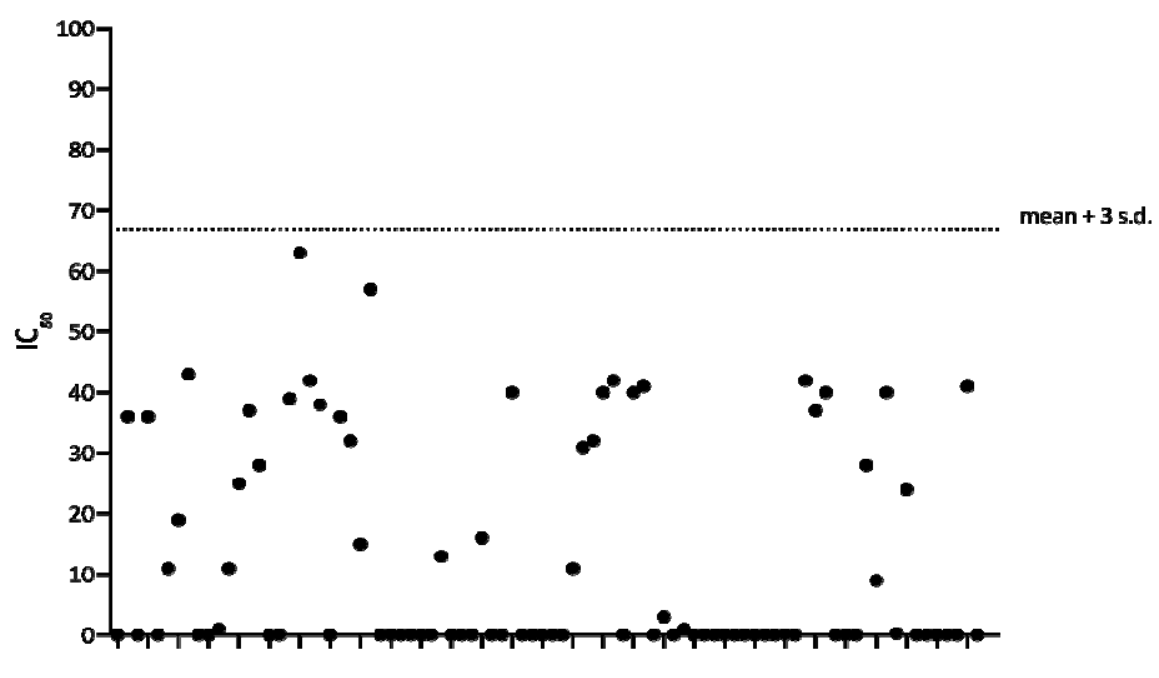

runs of the assay

Extended Data Fig. 4. PVNT cut-off determination for positive samples. Samples included showed no significant reduction in luminescence compared to PV only samples (no serum). 46 tests did not yield an $\mathrm{IC}_{50}$. The remaining 40 tests were found to have a low range of $\mathrm{IC}_{50}$ titres, but showed no significant reduction in luminescence and therefore were included in the analysis. Mean, standard deviation (s.d.) and cut-off point calculated with Prism 8. 
Extended Data Table 1. In vitro infection of Lloviu virus on monkey and human cell lines. Cells were infected with MOI 0.01 of the virus isolate. DPI refers to days post-virus infection.

$\begin{array}{cccccc} & \text { VeroE6 } & \text { SH-SY5Y } & \text { HepG2 } & \text { HCC78 } & \text { HCT116 } \\ & \begin{array}{c}\text { Monkey kidney } \\ \text { cells }\end{array} & \begin{array}{c}\text { Human } \\ \text { neuroblastoma }\end{array} & \begin{array}{c}\text { Hepatocyte } \\ \text { carcinoma }\end{array} & \begin{array}{c}\text { Human lung } \\ \text { adenocarcinoma }\end{array} & \begin{array}{c}\text { Human colon } \\ \text { carcinoma }\end{array} \\ \begin{array}{c}\text { 10 DPI Ct value } \\ 10 \text { DPI }\end{array} & 24.03 & 27.33 & 24.99 & 26.15 & 27.47 \\ \begin{array}{c}\text { supernatant Ct } \\ \text { value }\end{array} & 21.5 & 24.36 & 22.55 & 23.11 & 24.87\end{array}$

Extended Data Fig. 5. Blind passage of Lloviu virus isolate and cytopathic effect on SuBK12-08 cells ten days post infection (DPI), passage 4. Panel A - mock-infected cells 10 DPI, Panel B - Lloviu virus-infected cells 10 DPI
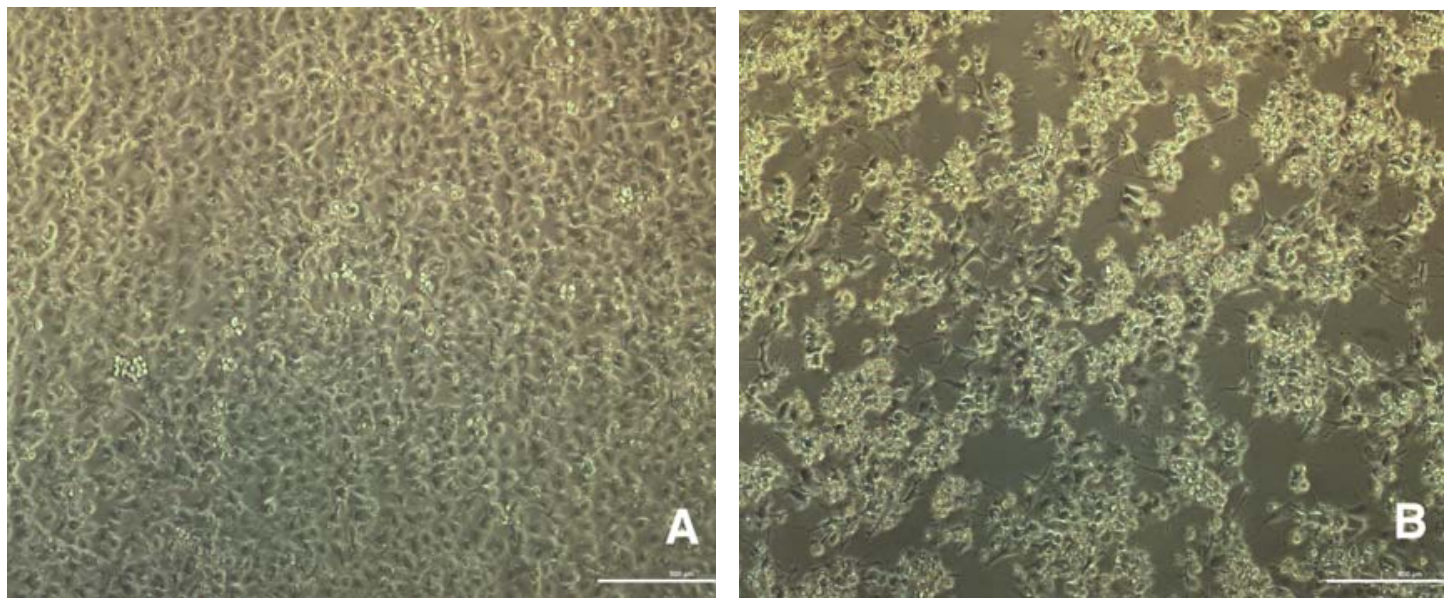

\section{References}

1. Burk, R. et al. Neglected filoviruses. FEMS Microbiol. Rev. 40, 494-519 (2016).

2. Malvy, D., McElroy, A. K., de Clerck, H., Günther, S. \& van Griensven, J. Ebola virus disease. The Lancet vol. 393 936-948 (2019).

3. Jombart, T. et al. The cost of insecurity: From flare-up to control of a major Ebola virus disease hotspot during the outbreak in the Democratic Republic of the Congo, 2019. Eurosurveillance 25, 1-4 (2020).

4. Ebola virus disease outbreak in Guinea, 2021.

https://www.ecdc.europa.eu/en/publications-data/ebola-virus-disease-outbreak-guinea- 
2021.

5. Leroy, E. M. et al. Fruit bats as reservoirs of Ebola virus. Nature 438, 575-576 (2005).

6. Towner, J. S. et al. Isolation of genetically diverse Marburg viruses from Egyptian fruit bats. PLoS Pathog. 5, e1000536 (2009).

7. He, B. et al. Filovirus RNA in Fruit Bats, China. Emerging infectious diseases vol. 21 1675-1677 (2015).

8. Yang, X.-L. et al. Genetically Diverse Filoviruses in Rousettus and Eonycteris spp. Bats, China, 2009 and 2015. Emerg. Infect. Dis. 23, 482-486 (2017).

9. Shi, M. et al. The evolutionary history of vertebrate RNA viruses. Nature 556, 197$202(2018)$.

10. Yang, X. Lou et al. Characterization of a filovirus (Měnglà virus) from Rousettus bats in China. Nature Microbiology vol. 4 390-395 (2019).

11. Goldstein, T. et al. The discovery of Bombali virus adds further support for bats as hosts of ebolaviruses. Nat. Microbiol. 3, 1084-1089 (2018).

12. Forbes, K. M. et al. Bombali Virus in Mops condylurus Bat, Kenya. Emerg. Infect. Dis. 25, 955-957 (2019).

13. Karan, L. S. et al. Bombali virus in Mops condylurus bats, Guinea. Emerg. Infect. Dis. 25, 1774-1775 (2019).

14. Negredo, A. et al. Discovery of an ebolavirus-like filovirus in europe. PLoS Pathog. 7, e1002304 (2011).

15. Kemenesi, G. et al. Re-emergence of Lloviu virus in Miniopterus schreibersii bats, Hungary, 2016 correspondence. Emerg. Microbes Infect. 7, (2018).

16. De Arellano, E. R. et al. First evidence of antibodies against lloviu virus in schreiber's bent-winged insectivorous bats demonstrate a wide circulation of the virus in spain.

Viruses 11, (2019).

17. Brinkmann, C. et al. The Tetherin Antagonism of the Ebola Virus Glycoprotein Requires an Intact Receptor-Binding Domain and Can Be Blocked by GP1-Specific Antibodies. J. Virol. 90, 11075-11086 (2016). 
18. Maruyama, J. et al. Characterization of the Envelope Glycoprotein of a Novel Filovirus, Lloviu Virus. J. Virol. 88, 99-109 (2014).

19. Ng, M. et al. Cell entry by a novel European filovirus requires host endosomal cysteine proteases and Niemann-Pick \{C1\}. Virology 468-470, 637-646 (2014).

20. Manhart, W. A. et al. A Chimeric Lloviu Virus Minigenome System Reveals that the Bat-Derived Filovirus Replicates More Similarly to Ebolaviruses than Marburgviruses. Cell Rep. 24, 2573-2580.e4 (2018).

21. Hume, A. J. et al. Recombinant Lloviu virus as a model to study inaccessible zoonotic viruses. bioRxiv 2021.08.02.454777 (2021) doi:10.1101/2021.08.02.454777.

22. Gryseels, S. et al. Role of Wildlife in Emergence of Ebola Virus in Kaigbono (Likati), Democratic Republic of the Congo, 2017. Emerg. Infect. Dis. 26, (2020).

23. Amman, B. R. et al. Seasonal Pulses of Marburg Virus Circulation in Juvenile Rousettus aegyptiacus Bats Coincide with Periods of Increased Risk of Human Infection. PLoS Pathog. 8, e1002877 (2012).

24. Hayman, D. T. S. Biannual birth pulses allow filoviruses to persist in bat populations. Proceedings. Biol. Sci. 282, 20142591 (2015).

25. Breman, J. G. et al. A search for Ebola virus in animals in the Democratic Republic of the Congo and Cameroon: ecologic, virologic, and serologic surveys, 1979-1980. Ebola Virus Study Teams. J. Infect. Dis. 179 Suppl, S139--47 (1999).

26. Leirs, H. et al. Search for the Ebola Virus Reservoir in Kikwit, Democratic Republic of the Congo: Reflections on a Vertebrate Collection. J. Infect. Dis. 179, S155-S163 (1999).

27. Reiter, P. et al. Field Investigations of an Outbreak of Ebola Hemorrhagic Fever, Kikwit, Democratic Republic of the Congo, 1995: Arthropod Studies. J. Infect. Dis. 179, S148-S154 (1999).

28. Szentiványi, T., Christe, P. \& Glaizot, O. Bat Flies and Their Microparasites: Current Knowledge and Distribution. Front. Vet. Sci. 6, 115 (2019).

29. Dick, C. W. \& Patterson, B. D. Bat flies: Obligate ectoparasites of bats. in Micromammals and Macroparasites: From Evolutionary Ecology to Management 
179-194 (Springer Japan, 2006). doi:10.1007/978-4-431-36025-4_11.

30. Reeves, W. K. \& Lloyd, J. E. Louse flies, keds, and bat flies (hippoboscoidea). in Medical and Veterinary Entomology 421-438 (Elsevier, 2018). doi:10.1016/B978-012-814043-7.00020-0.

31. Szentiványi, T., Estók, P. \& Földvári, M. Checklist of host associations of European bat flies (Diptera: Nycteribiidae, Streblidae). Zootaxa 4205, 101-126 (2016).

32. Quick, J. et al. Real-time, portable genome sequencing for Ebola surveillance. Nature 530, 228-232 (2016).

33. Quick, J. et al. Multiplex PCR method for MinION and Illumina sequencing of Zika and other virus genomes directly from clinical samples. Nat. Protoc. 12, 1261-1266 (2017).

34. Y, T. et al. Niemann-Pick C1 Heterogeneity of Bat Cells Controls Filovirus Tropism. Cell Rep. 30, 308-319.e5 (2020).

35. Tyson, J. R. et al. Improvements to the ARTIC multiplex PCR method for SARS-CoV2 genome sequencing using nanopore. bioRxiv vol. 31 (2020).

36. Lloviu cuevavirus sequencing protocol. https://www.protocols.io/view/lloviucuevavirus-sequencing-protocol-bmz3k78n.html.

37. Mandl, J. N. et al. Reservoir Host Immune Responses to Emerging Zoonotic Viruses. Cell 160, 20-35 (2015).

38. Jones, M. E. B. et al. Clinical, Histopathologic, and Immunohistochemical Characterization of Experimental Marburg Virus Infection in A Natural Reservoir Host, the Egyptian Rousette Bat (Rousettus aegyptiacus). Viruses 11, 214 (2019).

39. Caron, A. et al. Ebola virus maintenance: if not (Only) bats, what else? Viruses 10, (2018).

40. Hayman, D. T. S. As the bat flies. Science (80-. ). 354, 1099-1100 (2016).

41. Amman, B. R. et al. Marburgvirus Resurgence in Kitaka Mine Bat Population after Extermination Attempts, Uganda. Emerg. Infect. Dis. 20, 1761-1764 (2014).

42. Lourenço, S. \& Palmeirim, J. M. Which factors regulate the reproduction of 
ectoparasites of temperate-zone cave-dwelling bats? Parasitol. Res. 104, 127-134 (2008).

43. Turell, M. J., Bressler, D. S. \& Rossi, C. A. Short report: Lack of virus replication in arthropods after intrathoracic inoculation of Ebola Reston virus. Am. J. Trop. Med. Hyg. 55, 89-90 (1996).

44. Schuh, A. J. et al. No evidence for the involvement of the argasid tick Ornithodoros faini in the enzootic maintenance of marburgvirus within Egyptian rousette bats Rousettus aegyptiacus. Parasites and Vectors 9, (2016).

45. Szentiványi, T. et al. Host conservation through their parasites: Molecular surveillance of vector-borne microorganisms in bats using ectoparasitic bat flies. Parasite 27, (2020).

46. Jun, S. R. et al. Ebolavirus comparative genomics. FEMS Microbiology Reviews vol. $39764-778(2015)$.

47. Towner, J. S. et al. Marburgvirus Genomics and Association with a Large Hemorrhagic Fever Outbreak in Angola. J. Virol. 80, 6497-6516 (2006).

48. Dietz, C. Illustrated identification key to the bats of Europe. (2004).

49. Theodor, O. \& Theodor, O. illustrated catalogue of the Rothschild collection of Nycteribiidae (Diptera) in the British Museum (Natural History); with keys and short descriptions for the identification of subfamilies, genera, species and subspecies. (1967).

50. HILLYARD, P. D. Ticks of North-west Europe. (Field Studies Council, for the Linnean Society of London and the Estuarine and Coastal Sciences Association, 1996).

51. Mather, S. T., Wright, E., Scott, S. D. \& Temperton, N. J. Lyophilisation of influenza, rabies and Marburg lentiviral pseudotype viruses for the development and distribution of a neutralisation -assay-based diagnostic kit. J. Virol. Methods 210, 51-58 (2014).

52. Scott $\dagger$, S., Molesti, E. \& Temperton, N. The use of equine influenza pseudotypes for serological screening. J. Mol. Genet. Med. 06, 304-308 (2012).

53. REED, L. J. \& MUENCH, H. A SIMPLE METHOD OF ESTIMATING FIFTY PER CENT ENDPOINTS12. Am. J. Epidemiol. 27, 493-497 (1938). 
54. Ferrara, F. \& Temperton, N. Pseudotype neutralization assays: From laboratory bench to data analysis. Methods Protoc. 1, 1-16 (2018).

55. Jacobson, R. H. Validation of serological assays for diagnosis of infectious diseases. Rev. sci. tech. Off. int. Epiz vol. 17 (1998).

56. Lester, S. et al. Middle East respiratory coronavirus (MERS-CoV) spike (S) protein vesicular stomatitis virus pseudoparticle neutralization assays offer a reliable alternative to the conventional neutralization assay in human seroepidemiological studies. Access Microbiol. 1, e000057 (2019).

57. Nie, J. et al. Establishment and validation of a pseudovirus neutralization assay for SARS-CoV-2. Emerg. Microbes Infect. 9, 680-686 (2020).

58. Quick, J. Ebola virus sequencing protocol. (2019) doi:10.17504/protocols.io.7nwhmfe.

59. Quick, J. Forked from Ebola virus sequencing protocol. (2020) doi:10.17504/protocols.io.bbmuik6w.

60. Li, H. Minimap2: Pairwise alignment for nucleotide sequences. Bioinformatics 34, 3094-3100 (2018).

61. N, C.-N. et al. Modular approach to customise sample preparation procedures for viral metagenomics: a reproducible protocol for virome analysis. Sci. Rep. 5, (2015).

62. Chrzastek, K. et al. Use of Sequence-Independent, Single-Primer-Amplification (SISPA) for rapid detection, identification, and characterization of avian RNA viruses. Virology 509, 159-166 (2017).

63. Suchard, M. A. et al. Bayesian phylogenetic and phylodynamic data integration using BEAST 1.10. Virus Evol. 4, (2018). 


\section{Acknowledgements}

The project was supported by the National Research, Development and Innovation Office (grant numbers: NKFIH FK131465, KH129599). G.K. was supported by the János Bolyai Research Scholarship of the Hungarian Academy of Sciences. G.E.T and Z.L. were supported by the Biological and Sportbiological Doctoral School of the University of Pécs, Hungary. The SuBK12-08 cell line was obtained from the scientists of Hokkaido University Center for Zoonosis Control and the University of Zambia. The authors wish to thank professor Ayato Takada for sharing the SuBK12-08 cells.

\section{Data Availability}

Genome sequences are available on NCBI GenBank: MW775010- MW775011 and MZ541881. Sequencing protocol and materials are listed here: https://www.protocols.io/view/lloviu-cuevavirus-sequencing-protocol-bmz3k78n.html

\section{Contributions}

G.K., G.E.T., M.M.N., S.S., B.Z., Z.L., Á.N, C.I.P., G.D., F.F. conceived the laboratory work. G.K., G.E.T., S.A.B., T.G., P.E., Z.L., Á.N., C.I.P., G.D., K.K. conducted field work. M.M.N., S.S., N.T., E.W. designed and conducted the neutralization experiments. S.A.B., T.G., P.E. handling of bats during fieldwork. P.E. ectoparasite identification. G.K., M.M., F.F. conceived the in vitro experiments. G.E.T., B.A.S., A.J.H., S.Z., G.K., P.M, B.V. sequencing and genomic analysis. B.A.S., M.M.N. visualization of results. G.K., F.J., S.S. conceptualization and supervision. G.K. wrote the manuscript. G.E.T., M.M.N., S.S., N.T., E.W., S.A.B., T.G., Á.N., F.J., A.J.H., E.M. edited the manuscript with contributions from all other authors. G.K., S.S., E.M., Á.N., F.J. provided supervision. All authors approved the manuscript.

Supplementary Information is available for this paper.

Correspondence and requests for materials should be addressed to G.K.

The authors have declared no competing interest. 
bioRxiv preprint doi: https://doi.org/10.1101/2021.08.10.455806; this version posted August 10,2021 . The copyright holder for this preprint (which was not certified by peer review) is the author/funder, who has granted bioRxiv a license to display the preprint in perpetuity. It is made available under aCC-BY-NC-ND 4.0 International license.

Table 1. PVNT results of seropositive bats paired with RT-PCR results

\begin{tabular}{|c|c|c|c|c|c|c|c|}
\hline Animal ID & $\begin{array}{c}\text { PVNT } \\
\text { LLOV } 1 \\
\text { IC }_{50}\end{array}$ & $\begin{array}{c}\text { PVNT LLOV } \\
2 \text { IC }_{50}\end{array}$ & Mean $\mathrm{IC}_{50}$ & Serology & Real-time RT-PCR & Status & $\begin{array}{l}\text { Collection } \\
\text { Date }\end{array}$ \\
\hline LLOV 98 & 211 & $*$ & 211 & pos & N/A & Alive & 2018.09.18. \\
\hline LLOV 99 & 156 & 174 & 165 & pos & N/A & Alive & 2018.09.18. \\
\hline LLOV 102 & 132 & $*$ & 132 & pos & neg & Alive & 2018.09.18. \\
\hline LLOV 110 & 64 & $*$ & $64 * *$ & pos & neg & Alive & 2018.09.18. \\
\hline LLOV 115 & 143 & 197 & 170 & pos & N/A & Alive & 2018.09.18. \\
\hline LLOV 118 & 155 & $*$ & 155 & pos & neg & Alive & 2018.09.18. \\
\hline LLOV 130 & 177 & 59 & 118 & pos & neg & Alive & 2018.09.18. \\
\hline LLOV 138 & 83 & $*$ & 83 & pos & neg & Alive & 2018.09.18. \\
\hline LLOV 143 & 90 & $*$ & 90 & pos & neg & Alive & 2018.09.18. \\
\hline LLOV $1^{\dagger}$ & 768 & N/A & 768 & pos & pos (lung, spleen) & Dead & 2016.02 .11 . \\
\hline LLOV $2^{\dagger}$ & 2,999 & 3,972 & 3,485 & pos & neg & Dead & 2016.02 .11 . \\
\hline LLOV $169^{\dagger}$ & 172 & 337 & 254 & pos & pos (lung, spleen) & Dead & 2019.01.31. \\
\hline LLOV $170^{\dagger}$ & 757 & 1,136 & 946 & pos & neg & Dead & 2019.01.31. \\
\hline Control 1 & non-neut. & non-neut. & & neg & neg & Alive & 2019.08.08. \\
\hline Control 2 & non-neut. & non-neut. & & neg & neg & Alive & 2019.08.08. \\
\hline
\end{tabular}

* - PVNT not performed due to lack of sufficient serum for a repeat experiment; $* *$ - Borderline positive value; ${ }^{\dagger}$ - dead at the original site of virus emergence, Northeast Hungary, Zemplén Mt.; Control 1 (Myotis myotis) and Control 2 (M. schreibersii) were used as healthy negative control animals from a distant bat roost site in Southwestern Hungary. 
bioRxiv preprint doi: https://doi.org/10.1101/2021.08.10.455806: this version posted August 10,2021. The copyright holder for this preprint (which was not certified by peer review) is the author/funder, who has granted bioRxiv a license to display the preprint in perpetuity. It is made available under aCC-BY-NC-ND 4.0 International license.

Table 2. Detailed information about LLOV RNA positive samples

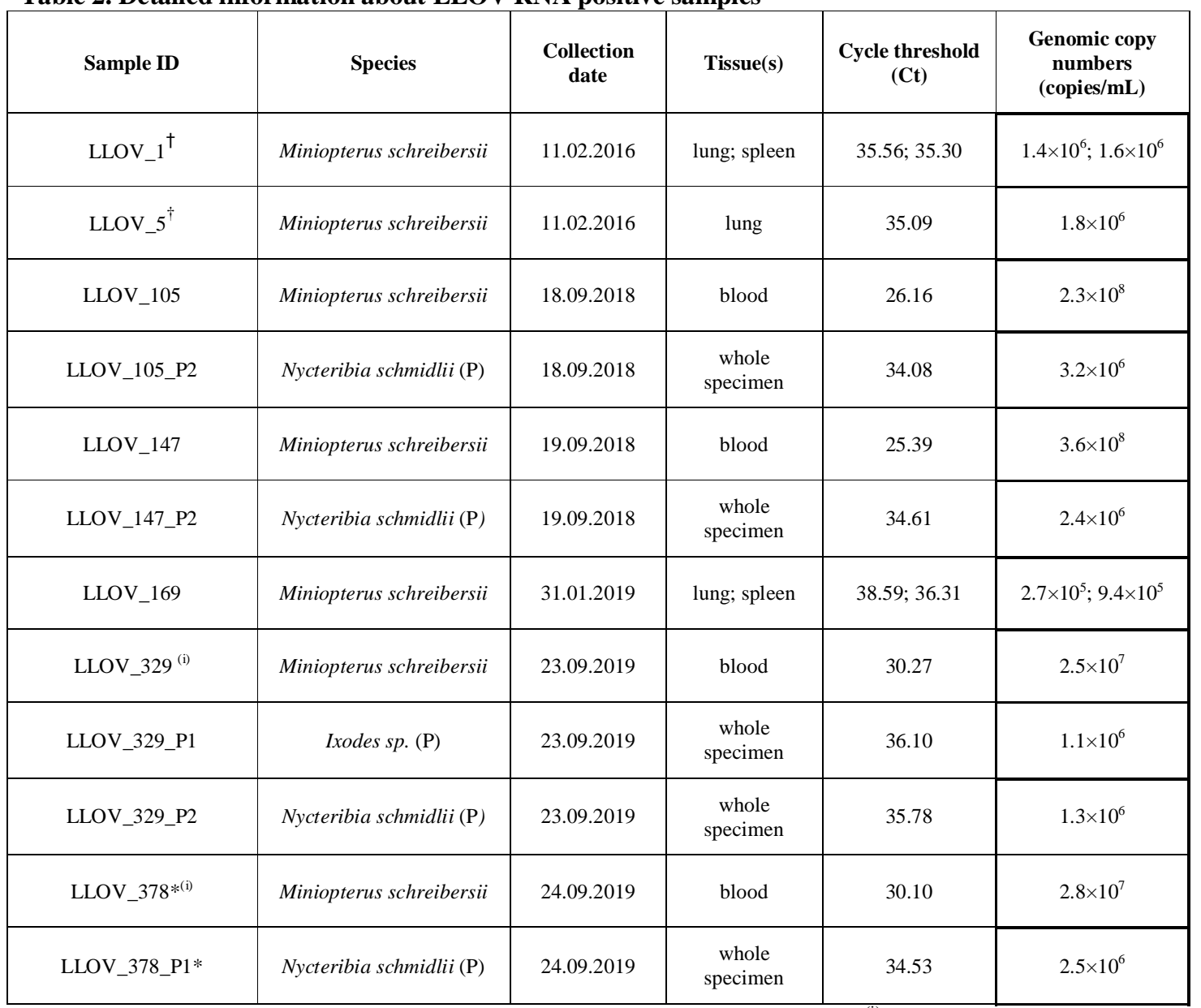

(P) - ectoparasite; * samples were subjected to viral genomic sequencing; ${ }^{(i)}$ sample was used for in vitro isolation efforts; ${ }^{\dagger}$ carcasses 